\title{
Gallimard
}

\section{ON NOMME LITTÉRATURE LA FRAGILITÉ DE L'HISTOIRE}

\section{Patrick Boucheron}

Gallimard | « Le Débat »

$2011 / 3 n^{\circ} 165$ | pages 41 à 56

ISSN 0246-2346

ISBN 9782070134144

Article disponible en ligne à l'adresse :

https://www.cairn.info/revue-le-debat-2011-3-page-41.htm

Distribution électronique Cairn.info pour Gallimard.

(C) Gallimard. Tous droits réservés pour tous pays.

La reproduction ou représentation de cet article, notamment par photocopie, n'est autorisée que dans les limites des conditions générales d'utilisation du site ou, le cas échéant, des conditions générales de la licence souscrite par votre établissement. Toute autre reproduction ou représentation, en tout ou partie, sous quelque forme et de quelque manière que ce soit, est interdite sauf accord préalable et écrit de l'éditeur, en dehors des cas prévus par la législation en vigueur en France. Il est précisé que son stockage dans une base de données est également interdit. 


\section{On nomme littérature la fragilité de l'histoire}

La littérature en impose à l'histoire. Quelquesuns parmi les historiens s'en réjouissent, d'autres bien plus nombreux s'en alarment - mais c'est ainsi : le bruit court qu'il appartient désormais au roman d'assumer une part de la vérité du passé. Un roman documenté, entendons-nous bien : les écrivains sont priés d'abandonner leur mauvais penchant à se complaire dans un formalisme futile pour se coltiner aux rugosités du réel. Quant aux historiens, ils seront invités à livrer leur expertise sur la vraisemblance des faits, dès lors qu'un roman plantant sa prose dans le petit lopin où se cultive leur réputation de compétence rencontre quelque succès. Dans tous les cas, la discipline historique est ramenée au rang de science auxiliaire de l'invention romanesque. On ne lui demande rien d'autre que d'accumuler des fiches afin que des écrivains véritables, plus imaginatifs et plus lestes, libres surtout de tout scrupule et de toute entrave, échafaudent des intrigues.

Telle est la petite fable que l'on nous livre aujourd'hui. Elle ramasse en une pelote serrée plusieurs fils noués qu'il n'est pas si aisé de démêler. Le premier, sans doute, a trait à l'histoire littéraire immédiate. Les noces de la littérature et du réel sont régulièrement célébrées comme d'heureuses retrouvailles, après un moment de repli minimaliste ou intimiste - dont la minceur autofictive ne serait que la petite monnaie. Rupture, donc, avec une littérature d'après guerre d'inspiration blanchotienne qui aurait fait d'écrire un verbe intransitif, la seule intrigue romanesque digne de la littérature étant désormais les aventures de la langue elle-même. Une telle chronologie pourrait être discutée. Elle n'en conforme pas moins aujourd'hui l'horizon de réception des œuvres, maintenant l'écrivain qui aspire au succès public dans un fâcheux dilemme quant au choix de son sujet: soit un grand nom dans les hauts vents de l'histoire (si possible la plus tragique), soit sa petite personne dans les clapotis de l'actualité (si possible la plus insignifiante). 
Patrick Boucheron

On nomme littérature

la fragilité de l'histoire
Ce n'est pas d'hier, pourtant, que la littérature entend dévoiler du même geste l'intensité du moi et l'immensité du monde - car c'est précisément cette croyance sociale qui la constitue en littérature. Le roman, notamment, naît (et renaît sans cesse) de sa volonté farouche de dire le vrai sur notre temps. Et sa vérité, parce qu'elle frappe l'imagination et s'inscrit dans l'expérience vécue du lecteur, est d'une essence supérieure à celle qu'établit péniblement l'historien, qui n'est rien d'autre qu'une exactitude vétilleuse. Lorsqu'un écrivain s'empare des choses passées, c'est moins pour témoigner de ce qui s'est passé que pour rendre intelligible et sensible ce que c'est, pour le temps, de passer. À l'aune de cette puissance de la littérature se mesure la tentation littéraire de l'historien. Car ce dernier partage avec l'écrivain bien plus que le goût, ou la nécessité, d'écrire sur le passé, pour l'éclairer comme passé et le rendre accueillant aux sollicitations de l'aujourd'hui. Parce qu'ils cherchent à rendre, par le travail propre des intrigues de la langue, les accrocs de leur texture, ils peuvent être, l'un comme l'autre, des artisans de la fragilité des temps.

\section{La littérature comme force supplétive de l'histoire}

Dans Robespierre, derniers temps, Jean-Philippe Domecq confiait à la forme littéraire le soin de rendre raison à ce qui, selon lui, se dérobait à l'histoire - le mystère de la résignation finale de Robespierre face aux thermidoriens. Revenant tout récemment sur cette expérience d'écriture hissant l'intuition au-dessus des modes ordinaires de compréhension historique, il a tenu à s'écarter de la démarche, au fond plus tradition- nelle, de l'intrusion romanesque qui consiste à combler de fiction les vides de l'histoire: "Croyant enrichir l'un par l'autre, on perd sur les deux tableaux: la fiction son crédit et le savoir ses preuves ${ }^{1}$." En examinant tour à tour (et de manière assez vindicative) Les Bienveillantes de Jonathan Littell (2006), Fan Karski de Yannick Haenel (2009), HHhH de Laurent Binet (2010), Domecq consacre l'importance de ce récent triplé romanesque comme le passage obligé du moment pour quiconque entend réfléchir aux rapports entre histoire et littérature. Cette réduction n'est rien d'autre qu'un effet du discours critique sur les œuvres lorsqu'il est soumis au tempo médiatique qui s'impose à la critique. Elle échappe pour une large part au contrôle, aux intentions, et sans doute à la compréhension des trois auteurs en question; mais c'est bien ainsi qu'il faut l'envisager.

Tout partirait donc de l'étonnant succès public et critique des Bienveillantes qui fit, en 2006, trembler la frontière entre fiction et histoire. Rappelons seulement que ce roman décrivait, avec une minutie obsessionnelle que certains lecteurs pouvaient juger complaisante, l'appareil d'État nazi à partir du point de vue d'un bourreau imaginaire, mais que l'auteur présentait comme vraisemblable. On le considéra bientôt comme le livre sur la Shoah. Toute l'affaire résidait donc dans cette prise de relais du devoir de mémoire par la fiction, qui, notons-le au passage, s'était parallèlement jouée sur le terrain cinématographique. Les historiens étaient alors sommés de répondre à cette injonction : pourquoi n'avaient-ils pas eux-mêmes écrit ce livre? N'étaient-ils donc plus capables de s'adresser à

1. Jean-Philippe Domecq, "La littérature comme acupuncture", dans Robespierre, derniers temps [1984], rééd. Gallimard ("Folio»), 2011, pp. 349-429, ici p. 367. 
Patrick Boucheron

On nomme littérature la fragilité de l'histoire tous? De là d'inévitables raidissements. Car le reproche était certainement injuste, y compris sur le plan littéraire, les grands chefs-d'œuvre de l'historiographie de la Shoah (ceux de Raul Hilberg, Saul Friedländer, Christopher Browning notamment) se caractérisant précisément par leur audace narrative et leur beauté formelle ${ }^{2}$. Raul Hilberg le dit très bien, lorsqu'il se justifie d'avoir composé La Destruction des fuifs d'Europe en s'inspirant de principes d'harmonie musicale : «Seul un artiste consommé peut recréer ce fait $^{3}$."

Yannick Haenel concevait sans doute l'écriture de son fan Karski comme une réponse à Littell, coupable, selon lui, d'avoir donné forme au nihilisme contemporain en esthétisant la barbarie; les remous provoqués par sa parution constituaient d'une certaine manière une réplique de cette secousse initiale. L'écrivain tentait d'y protéger une transgression majeure (donner pour titre à un roman le nom propre d'un personnage historique) avec un dispositif narratif qui visait à protéger un strict partage entre le réel «documentaire» et l'invention littéraire qu'il qualifie de "fiction intuitive». Il ne résista pas aux assauts de la critique, qui faisait à son livre un procès en inexactitude. En tentant de chroniquer l'affaire qui me semblait symptomatique d'un embarras contemporain sur la mémoire, mais aussi sur la confiance que l'on pouvait encore accorder aux formes académiques de l'écriture de l'histoire, je courais après les rebondissements d'une polémique qui radicalisait les positions de chacun : l'Historien défendant le fait vrai, l'Écrivain l'éternelle vérité de la littérature ${ }^{4}$. $\mathrm{Au}$ moment où l'article paraissait, un autre roman mettait en scène cette hésitation, et en faisait même le ressort de l'intrigue $-\mathrm{HHhH}$ de Laurent Binet.

L'auteur y entrelace trois fils narratifs : celui de la biographie de Reinhard Heydrich, homme clé de l'appareil d'État nazi dont on disait qu'il était le cerveau de Himmler (Himmlers Hirn heißt Heydrich); celui du roman d'aventures de deux soldats tchécoslovaques qui ont entrepris de tuer la "Bête Blonde»; celui du narrateur qui ne sait comment raconter cette histoire. Suis-je autorisé à fabuler? «Non, ce n'est pas inventé. Quel intérêt d'ailleurs y aurait-il à inventer du nazisme ${ }^{5}$ ?" Peu importent les qualités littéraires de ce roman : il fut remarqué, et de même que le fan Karski ne témoignait pas de ce qui s'était passé en 1942 mais de ce qui se passe aujourd'hui lorsqu'on évoque 1942, de même le roman de Laurent Binet témoigne bien d'un nouvel état de la mémoire contemporaine sur la Seconde Guerre mondiale. On peut le juger déplaisant, et il l'est sans doute, tant il exprime les travers d'une époque qui fait assaut de bonne conscience en feignant de croire que tous ceux qui ne furent ni des victimes ni des héros ne pouvaient être que des bourreaux. Mais c'est ainsi : au moins aura-t-il répondu à l'une des attentes de la littérature aujourd'hui, qui est peut-être de déconcerter notre propre rapport au temps.

2. Florent Brayard, «La longue fréquentation des morts. À propos de Browning, Kershaw, Friedländer - et Hilberg", Annales. Histoire, sciences sociales, $\mathrm{n}^{\circ} 64-5$, septembre-octobre 2009, pp. 1053-1090.

3. Raul Hilberg, La Politique de la mémoire [1994], trad. de l'anglais, Gallimard, 1996, p. 79. Je souligne.

4. Patrick Boucheron, "'Toute littérature est assaut contre la frontière." Note sur les embarras historiens d'une rentrée littéraire", Annales. Histoire, sciences sociales, $\mathrm{n}^{\circ}$ 65-2, mars-avril 2010, pp. 441-467.

5. Laurent Binet, $\mathrm{HHhH}$, Grasset, 2010, p. 69. 
Patrick Boucheron

On nomme littérature

la fragilité de l'histoire
Un malentendu et trois réductions

Telle est donc la manière dont on présente habituellement la reconfiguration actuelle entre la littérature et l'histoire. On y aura aisément remarqué une triple inflexion. La première est la plus évidente : elle consiste à rabattre cette question sur le rapport entre récit historien et fiction romanesque. Or, si le roman n'est pas le tout de la littérature, la question de la vérité n'épuise pas, loin s'en faut, l'ensemble des problèmes que pose l'opération historiographique. Que l'on en défende les droits ou que l'on en dénonce les dangers, dès lors que l'on pose la littérature comme un récit fictionnel libérant du relevé factuel de l'histoire, on s'expose à bien des naïvetés. Sur l'écriture de l'histoire, bien entendu, dont on sait depuis longtemps - depuis toujours? - qu'"elle ne saurait se confondre avec les événements dont elle fournit la représentation ${ }^{6}$ " et n'établit par conséquent aucun rapport transparent avec ce qui fut réellement. Sur la littérature également, dont il n'est guère difficile de comprendre combien elle a puisé dans les sciences de l'homme, ses motifs et ses méthodes, de quoi desserrer l'étreinte romanesque et hybrider les formes littéraires de son appréhension du réel - retrouvant ainsi ce que Gérard Macé appelle, en lisant les anthropologues (ou, plus précisément, ce qu'il y a d'inévitablement littéraire dans le discours des anthropologues), le goût de l'homme? .

Parce qu'il fut chaviré en 1978 par la lecture du Mémorial de la déportation des fuifs de France de Serge Klarsfeld, Patrick Modiano a, de son propre aveu, "douté de la littérature». C'est peut-être pour en sauver la dignité qu'il fit, dans Dora Bruder, un usage presque ascétique de la notation d'archive, noms et dates parsemant sèchement son texte comme des cailloux jetés contre l'oubli, ralentissant presque cérémonieusement le rythme de sa prose. Parce qu'«il faut longtemps pour que ressurgisse à la lumière ce qui a été effacé ${ }^{8}$ " Modiano installait la figure du romancier en archéologue de la mémoire, posture si commune aujourd'hui qu'elle peut être aisément contrefaite par des faiseurs sans scrupule. Reste que l'on ne peut feindre d'ignorer que c'est toujours ici l'histoire la plus tragique de la Seconde Guerre mondiale qui précipite la controverse opposant vérité historique et mensonge romanesque. Ce qui fait scandale est bien, selon l'expression de Laurent Binet, d'inventer du nazisme. Tout le reste est permis : personne ne songe à s'indigner que Pierre Michon invente dans Les Onze une page de Michelet décrivant un tableau qui n'existe pas. Interrogé sur cette tolérance au plus fort de l'affaire Jan Karski, Bruno Tessarech (qui, lui aussi, avait consacré un roman au résistant polonais) répondait avec candeur que l'écrivain ne devait au fond manifester de "respect» que lorsqu'il s'agissait de "sujets importants" et de personnages "qui ont eu une place cruciale dans l'histoire $"^{9}$. Il est vrai que Pierre Michon ne traitait que de Robespierre et de la Terreur... Mais l'affirmation vaut symptôme: ce que l'on nomme histoire dans les débats qui concernent ses frontières tremblantes avec la littérature se réduit le plus souvent, explicitement ou non, à la mémoire des

6. Jean Levi, La Chine romanesque : fictions d'Orient et d'Occident, Éd. du Seuil, 1995, p. 131.

7 . 2002

7. Gérard Macé, Le Goût de l'Homme, Le Promeneur, p. 15 .

8. Patrick Modiano, Dora Bruder, Gallimard, 1997,

9. Bruno Tessarech (auteur de Les Sentinelles, Grasset, 2009), interrogé par Emmanuel Laurentin dans "La fabrique de l'histoire", France Culture, 9 décembre 2009. 
Patrick Boucheron
On nomme littérature
la fragilité de l'histoire violences du xxe siècle, et tout particulièrement à la Shoah.

Que celle-ci impose, comme un devoir moral, sinon de repousser, du moins de contrôler strictement la tentation fictionnelle est devenu une évidence, dès lors que le danger négationniste n'est pas, loin s'en faut, écarté. Écrivant à Luce Giard combien L'Écriture de l'histoire de Michel de Certeau (1975) lui fut une lecture bouleversante ("Nous le savons désormais, l'historien écrit, il produit le lieu et le temps, mais il est luimême dans un lieu et dans un temps"), Pierre Vidal-Naquet se résignait pourtant à "se raccrocher à cette vieillerie, "le réel", "ce qui s'est authentiquement passé", comme disait Ranke au siècle dernier", car tant que sévissaient les assassins de la mémoire, mieux valait "distinguer le roman de l'histoire " ${ }^{10}$. L'histoire spécifique du négationnisme en France impose donc une troisième réduction au débat sur la littérature et l'histoire. Car celui-ci paraît bien incorrigiblement français - et c'est d'ailleurs dans l'histoire très française, sollersienne pourrait-on dire, d'une tradition littéraire hexagonale que campe crânement Yannick Haenel, qui se déclare «le frère de la Chartreuse et des Illuminations ${ }^{1{ }^{1}}$ ".

Peu de temps après son fan Karski paraissait la traduction de Hammerstein ou l'Intransigeance, impressionnant de calme et de profondeur, malgré sa forme éminemment composite. Hans Magnus Enzensberger y reconstituait la vie du général Kurt von Hammerstein, chef d'étatmajor de la Reichswehr, qui demanda en 1934 à être démis de ses fonctions pour ne pas avoir à obéir à Hitler. Enzensberger le faisait sur la base d'un important travail documentaire, parfaitement identifiable dans le cours d'un récit jalonné par sept "gloses» où l'auteur a cantonné ses jugements historiques et treize "conversations posthumes" dans lesquelles il renoue avec l'ancienne forme littéraire du «dialogue avec les morts" pour interroger les protagonistes sur le sens de leurs actions. Expliquant dans un post-scriptum "pourquoi ce livre n'est pas un roman", Enzensberger - aussi célèbre en Allemagne comme poète que comme essayiste ne se justifiait pas autrement que par un aveu de modestie face à la charge de l'histoire, si écrasante en vérité qu'elle ne devrait inspirer que des formes incertaines : "Que tout le monde, même un écrivain, fasse du mieux qu'il peut ${ }^{12}$."

Dans son usage subtil de la photographie, à la fois point d'appui documentaire et tremplin vers la rêverie, l'auteur de Hammerstein s'inscrit d'évidence dans une tradition sebaldienne - on pense notamment aux Anneaux de Saturne ${ }^{13}$. Écrivain de la hantise qui traque les revenances de l'histoire à la recherche de crimes oubliés ${ }^{14}$, W. G. Sebald est sans doute l'auteur européen qui a le plus contribué à inquiéter récemment la frontière entre histoire et littérature. Il y a du chasseur en lui, mais sous la forme aimable et nabokovienne du chasseur de papillons; du psychanalyste également (car ces papillons sont des souvenirs à saisir, puis à épingler sous forme de photographies); du policier, enfin, qui cherche à travers ses clichés à faire "apparaître les fantômes ${ }^{15}$ ". Sans doute est-ce aussi la démarche de Javier Cercas, qui avait jointoyé enquête

10. Pierre Vidal-Naquet, "Lettre», dans Luce Giard (sous la dir. de), Michel de Certeau, 1987 ("Cahiers pour un temps", centre Georges-Pompidou), pp. 71-72.

11. Yannick Haenel, Le Sens du calme, Mercure de France, 2011, p. 107.

12. Hans Magnus Enzensberger, Hammerstein ou l'Intransigeance. Une histoire allemande [2008], trad. de l'alld., Gallimard, 2010, pp. 349 et 363.

13. W. G. Sebald, Les Anneaux de Saturne [1995], trad. de l'alld., Gallimard, 2003.

14. Jean-François Hamel, Revenances de l'histoire. Répétition, narrativité, modernité, Minuit, 2006.

15. Muriel Pic, W. G. Sebald. L'image papillon, Presses du Réel, 2008, p. 61. 
Patrick Boucheron

On nomme littérature

la fragilité de l'histoire historique et construction romanesque dans le «récit réel» des Soldats de Salamine, faisant surgir les spectres de la guerre civile espagnole ${ }^{16}$. Mais il s'agissait alors de cerner les silences de mémoires affrontées; dans le cas de la tentative de coup d'État du 23 février 1981 que tout le monde a vue en direct à la télévision (ou, plus précisément, que tout le monde croit avoir vue), la mémoire est saturée par cet effet de sidération propre à l'impression télévisuelle, qui produit immédiatement une aura d'irréalité. C'est elle, vraisemblablement, qui met en échec la première version du livre, que l'auteur reconnaît avoir écrit comme un roman : "Étant incapable d'inventer ce que je sais de l'événement pour tenter d'en illuminer la réalité par la fiction, je me suis résigné à le raconter." L'histoire sert ici à "transformer l'échec de mon roman", mais elle a "l'insolence de ne renoncer à rien" - et surtout pas à son ambition littéraire ${ }^{17}$.

Inutile de multiplier les exemples : en élargissant la focale à d'autres expériences d'écriture, on comprend aisément combien le débat français actuel sur les droits du roman face au devoir de mémoire de la Seconde Guerre mondiale est à la fois oublieux des complexités de l'histoire et des diversités de la littérature. Car tout à sa puissance de séduction, celle-ci ne se laisse jamais intimider par les interdits qu'on lui oppose. A-t-on débattu du régime de vérité auquel prétend l'œuvre, profondément inconvenante, de James Ellroy? Il n'aspire pourtant à rien de moins qu'à révéler dans ses romans l'histoire secrète des États-Unis : Underworld USA clôt une trilogie qu'Ellroy appelle lui-même sa "carrière d'historien". Le narrateur y présente son livre comme le résultat d'un travail de surveillance ("J'ai suivi des gens", "Je suis resté dans l'ombre») lui permettant d'assembler «des rumeurs plausibles et des indiscrétions d'ini- tiés». Un "assemblage», donc, d'archives véritables - "ce livre est construit sur des documents publics détournés et des journaux intimes dérobés»-qui constitue une vision paranoïaque de l'histoire ( $"$ je suis venu vous dire que tout est vrai et que ce n'est pas du tout ce que vous pensez»), l'écrivain étant celui qui permet de découvrir "le reste de l'histoire ${ }^{18}$.

Ce qui reste aux historiens

Faut-il abandonner son reste à la littérature? Les historiens ne s'y montrent guère disposés, eux qui se veulent, selon le mot de Paul Veyne, les romanciers du vrai ${ }^{19}$. Aussi tentent-ils, face à l'offensive que l'on vient de dire, de garder la main. Une des manières de le faire est sans doute de redéfinir par eux-mêmes le savoir social dont la littérature serait porteuse, bien au-delà de son éventuelle valeur documentaire. Il s'agit moins alors de définir les spécificités d'une source que d'enrichir les potentialités d'une ressource, et de comprendre comment, à un moment donné, la société y puise ses valeurs et ses règles ${ }^{20}$. Ainsi peut-on, par exemple, reconstituer l'univers de lecture du roman balzacien durant la monarchie de Juillet à travers ses appropriations singulières, et, du même coup, comprendre comment la société elle-même -

16. Javier Cercas, Les Soldats de Salamine [2001], trad. de l'espagnol, Arles, Actes Sud, 2002, p. 79.

17. Id., Anatomie d'un instant [2009], trad. de l'espagnol, Arles, Actes Sud, 2010, pp. 21 et 22.

18. James Ellroy, Undeworld USA, trad. de l'anglais, Payot \& Rivages, 2010, p. 21. 1971.

19. Paul Veyne, Comment on écrit l'histoire, Éd. du Seuil,

20. "Savoirs de la littérature», Annales. Histoire, sciences sociales ( $\mathrm{n}^{\circ}$ 65-2, mars-avril 2010, sous la direction d'Étienne Anheim et Antoine Lilti). 
Patrick Boucheron

On nomme littérature

la fragilité de l'histoire

dans sa manière d'envisager la découpe de ses identités, la hiérarchie de ses grandeurs ou les allures de son régime d'historicité - est en partie produite par cette représentation romanesque ${ }^{21}$. Cela ne concerne d'ailleurs pas spécifiquement la littérature, mais également la création cinématographique au $\mathrm{xx}^{\mathrm{e}}$ siècle, en tant que l'une et l'autre sont des formes de l'art qui d'évidence en sait plus long sur nous que nous sur lui ${ }^{22}$. L'historien en prise sur la littérature doit en tout cas se garder à la fois du désenchantement positiviste et de l'exaltation esthétique, pour toujours s'interroger sur les effets de la catégorisation littéraire elle-même ${ }^{23}$.

Ainsi redéfinie, la portée strictement historique de la littérature déborde sa nature référentielle - et l'historien peut l'appréhender sereinement, sans pour autant lâcher sa casquette de "flic du référent", selon l'expression de Régine Robin ${ }^{24}$, couvre-chef auquel il tient tant, et sans doute à juste titre. Pour Carlo Ginzburg, «les romanciers font des découvertes techniques que les historiens peuvent utiliser comme des dispositifs cognitifs 25 ». C'est particulièrement vrai, sans doute, de la mise en intrigue de l'historicité elle-même, qui doit toujours bien plus à l'expérience littéraire qu'à l'opération historiographique. Est-ce à dire qu'il faudrait chercher, derrière le travail des historiens, les motifs et les modèles issus de la littérature de leur temps? Que la sédimentation braudélienne des temporalités, par exemple, ne serait rien d'autre que la démarcation dans le champ de l'histoire de ce qu'expérimentait le roman d'après guerre (chez John Dos Passos notamment)?

Cette idée appelle au moins deux objections. La première est relative à la mémoire des œuvres, dont l'effet peut toujours être différé, déplacé ou transformé, au gré des réinventions successives d'un patrimoine littéraire qui demeure ouvert aux appropriations intempestives ${ }^{26}$. Ce n'est pas nécessairement l'historiographie du dernier tiers du XIXe siècle qui se révèle la plus profondément flaubertienne, et c'est bien aux historiens d'aujourd'hui que l'écriture de Stendhal adresse un défi implicite, lorsqu'il mêle sa voix à celle de ses personnages en redressant l'italique et en faisant tomber les guillemets ${ }^{27}$. Arrêtonsnous un instant sur une expérience singulière et méconnue (du moins en France): celle du médiéviste italien Vito Fumagalli. Il usa avec audace et liberté de nombreux modèles littéraires pour tenter de reconstituer, en de courts textes intensément suggestifs, les paysages sensibles des temps barbares. Ainsi lorsqu'il prête l'oreille, mais avec cette écoute flottante que Michelet accordait aux archives, à une page de l'historien Jordanès qui évoque l'ultime rivage des Goths, cette Scanie d'au-delà des grandes forêts enneigés : prises par la morsure du gel, les îles se perdent dans une brillance sans limites dont l'immense clarté aveugle les loups affamés ${ }^{28}$. Mais lorsqu'il tente de caractériser l'orgueil nobiliaire, les sources médiévales ne lui suffisent plus : il tente de les éclairer par des contre-feux littéraires, jouant de l'anachronisme contrôlé.

21. Judith Lyon-Caen, La Lecture et la vie. Les usages $d u$ roman dans la France de Balzac, Tallandier, 2006. 2008.

22. Antoine de Baecque, L'Histoire-caméra, Gallimard,

23. Christian Jouhaud, Dinah Ribard et Nicolas Schapira, Histoire, Littérature, Témoignage. Écrire les malheurs du temps, Gallimard ("Folio»), 2009.

24. Régine Robin, "L'écriture à la trace. Entretien", Vacarme, $\mathrm{n}^{\circ} 54,2011$, pp. 5-12. p. 97.

25. Carlo Ginzburg, Un seul témoin, Bayard, 2007,

26. Judith Schlanger, La Mémoire des cuvres, Lagrasse, Verdier, 2008.

27. Carlo Ginzburg, Le Fil et les traces. Vrai faux fictif [2006], trad. de l'italien, Lagrasse, Verdier, 2010, pp. 249273.

28. Vito Fumagalli, Paysages de la peur. L'homme et la nature au Moyen Âge [1994], Bruxelles, Éd. de l'Ulb, 2009, pp. 26-27. 
Patrick Boucheron

On nomme littérature

la fragilité de l'histoire
Fumagalli fait quelques pas avec la cour de Saint-Simon, ne s'y attarde pas, puis s'arrête sur le baron de Charlus qui traîne son noble ennui dans Le Temps retrouvé. C'est, dit-il, le Marc Bloch de L'Étrange Défaite qui l'y mène, dans lequel il lit les résonances avec le «Moyen Âge proustien " qu'il veut camper ${ }^{29}$.

Ces résonances s'entendent bien comme des échos symétriques : ce fait empêche également d'envisager l'échange entre histoire et littérature comme un simple emprunt des motifs narratifs de l'une par l'autre. Ce qu'il y a de plus profondément historique dans la prose de Julien Gracq n'est ni l'inscription de ses récits dans un passé plus ou moins reconnaissable, ni même sa philosophie de l'histoire souvent explicitement formulée dans ses écrits critiques, mais la manière dont l'écrivain rend, par les moyens propres du roman, un certain régime du temps et de ses changements d'allure dans lequel la brusquerie de l'événement est longtemps différée et presque déjà remémorée avant même qu'il ait lieu. Or, comme l'a montré Étienne Anheim, cette "poétique d'un surgissement déjà advenu ${ }^{30}$ " s'épuise progressivement dans les années 1960, au moment où les historiens s'emparent à leur tour de cette question de l'événement, à la manière de Georges Duby dans Le Dimanche de Bouvines, c'est-à-dire en cherchant moins à la théoriser qu'à l'exprimer de manière sensible et concrète (littéraire, donc) au fil même de la narration. La question devient alors : l'écrivain en quête de la "transcription de l'histoire ${ }^{31}$ " peut-il se passer de lire les historiens, non comme réservoir de documentation mais bien comme source d'inspiration?

L'œuvre de Pierre Michon est sans doute la plus caractéristique aujourd'hui de ce compagnonnage resserré. Ce qui s'y joue n'est pas seulement un rapport au document, à l'érudi- tion, à la lettre, à la mémoire, à la vie même mais à tout cela en même temps, à tout ce qui peut encore donner sens au métier des historiens, si tant est qu'ils daignent se donner la peine. Alors verront-ils ce que Michon saisit dans la force somptueuse d'une écriture qui compose ces portraits "éblouissants et sauvages" dont a parlé Jean-Pierre Richard ${ }^{32}$. C'est Aetius, le vainqueur d'Attila aux champs Catalauniques en 451, qui écrit pour ralentir l'oubli, "parce qu'il engourdit déjà de ses brumes la vie d'Attalus, parce qu'on ment beaucoup, on ne sait déjà plus rien", parce qu'il faut rétablir la vérité d'un homme contre les chroniqueurs qui ne cessent de la travestir, qui ne savent déjà plus combien Attalus "aimait le sifflement calme des faux les matins de printemps ${ }^{33}$ ». C'est Barthélemy Prunières, paléontologue et poète, qui rêve de "rassembler chaque jour le puzzle infini de l'humanité morte ${ }^{34}$ " et qui, au moment de mourir, se souvient d'une de ses phrases abandonnées avec une désinvolture toute rimbaldienne dans une publication savante mais qui, décidément, sonnait bien et rassemblait donc d'un trait ce que le temps avait disjoint : «Tous ces os avaient été blanchis par la pluie, la rosée et la neige ${ }^{35}$." C'est Faulkner dans Corps du roi,

29. Ibid., p. 182.

30. Étienne Anheim, "Julien Gracq. L'œuvre de l'histoire", Annales. Histoire, sciences sociales, $\mathrm{n}^{\circ}$ 65-2, mars-avril 2010, pp. 377-416, ici p. 415.

31. Emmanuel Bouju, La Transcription de l'histoire. Essai sur le roman européen de la fin du vingtième siècle, Rennes, PUR, 2006.

32. Jean-Pierre Richard, Chemins de Michon, Lagrasse, Verdier, 2008, p. 8.

33. Pierre Michon, L'Empereur d'Occident, Fata Morgana, 1989, pp. 36 et 37 . p. 40 .

34. Id., Mythologies d'hiver, Lagrasse, Verdier, 1997,

35. Voir Caroline Callard, "Le poète et l'historien : un art du kidnapping", Critique, ${ }^{\circ} 694$, mars 2005, pp. 174186. 
Patrick Boucheron

On nomme littérature la fragilité de l'histoire c'est Michelet dans Les Onze, ce grand roman de la présence réelle de l'histoire dans les œuvres. Ruse suprême de la fiction, l'écrivain y fait la leçon à l'historien, lui reprochant de ne pas avoir décrit avec exactitude le tableau de Corentin, précisément parce qu'il l'a vu - et que cette vision emporte nécessairement avec elle des transpositions, des confusions, des réminiscences. Aussi faut-il à Pierre Michon inventer une page de l'Histoire de la Révolution française sur une peinture qui n'existe pas pour dire le vrai sur l'écriture de Michelet, sur ce moment très précis où «Michelet n'est plus le maître de sa fiction, cette fable si juste qui vient de sortir de son esprit l'enivre, l'emporte, et il l'enfourche sans ambages ${ }^{36}{ }^{\prime}$.

Des fictions qui ne mentent pas

Il existe donc un évident effet de symétrie entre deux questions qui, pour être strictement disjointes, n'en échangent pas moins leurs effets : la question des savoirs de l'écriture, dans lesquels les modes d'intelligibilité de l'histoire trouvent en partie leur origine, et la question de l'écriture des savoirs, donnant une dimension inévitablement littéraire à toute mise en récit historiographique. "Par conséquent, comme le remarque Jacques Rancière, pour que l'histoire puisse penser ce qu'elle fait, elle doit aussi s'interroger sur les origines littéraires de ses modes d'interprétation ${ }^{37}$." Une telle interrogation a, on le sait, longtemps produit de l'inquiétude. En décrivant l'homologie formelle entre le récit historien et la fiction romanesque, Hayden White a eu le mérite d'ébranler quelques-uns des préjugés scientistes qu'entraîne l'illusion référentielle ${ }^{38}$ - que l'on peut définir comme «ce dispositif d'écriture de la mimesis [par lequel le lecteur] croira avoir affaire à quelque chose du monde réel, qui se raconte tout seul, dans la transparence de la narration ${ }^{39} \%$. La manière dont le discours de l'historien assure sa propre scientificité et se démarque de l'invention fictionnelle (ou simplement du soupçon qui pèse sur la possible indistinction entre récit vrai et récit inventé) n'est rien d'autre en elle-même qu'un procédé narratif - c'est «l'ensemble des procédures littéraires par lesquelles un discours se soustrait à la littérature, se donne un statut de science et le signifie ${ }^{40}$ ». Bruno Latour ne dit pas autre chose dans le champ des sciences exactes, lorsqu'il décrit la rhétorique de la démonstration scientifique comme l'une des formes de l'éloquence, celle qu'Aristote appelait justement "l'éclat nécessaire au brillant de la vérité ${ }^{41}$ ».

Reste qu'un soupçon pèse, et qu'il pèse de plus en plus lourd, sur ce type de démarche, que l'on dira "narrativiste» pour mieux la disqualifier. Paul Ricœur redoutait déjà qu'elle «fasse oublier la sorte de contrainte que l'événement passé exerce sur le discours historique à travers les documents connus, en exigeant de celui-ci une rectification sans $\mathrm{fin}^{42}$ ». Contre ce qu'il appelle «l'attaque sceptique lancée à la scientificité des

36. Pierre Michon, Les Onze, Lagrasse, Verdier, 2009, p. 129.

37. Jacques Rancière, "Les hommes comme animaux littéraires" [1999], repris dans Et tant pis pour les gens fatigués. Entretiens, Ed. Amsterdam, 2009, p. 142.

38. Hayden White, Metahistory: The Historical Imagination in Nineteenth-Century Europe, Baltimore, Johns Hopkins UP, 1973.

39. Régine Robin, La Mémoire saturée, Stock, 2003, p. 289.

40. Jacques Rancière, Les Noms de l'Histoire. Essai de poétique du savoir, Éd. du Seuil, 1992, p. 21.

41. Bruno Latour, Cogitamus. Six lettres sur les humanités scientifiques, La Découverte, 2010, p. 100.

42. Paul Ricœur, Temps et récit, t. III, Éd. du Seuil, 1985, p. 280. 
Patrick Boucheron

On nomme littérature

la fragilité de l'histoire récits historiques ${ }^{43}$ ", Carlo Ginzburg réaffirme l'éthique de l'historien comme artisan de la preuve, qui ne doit renoncer ni à l'audace de l'interprétation, ni au tact qu'impose la manipulation des choses du passé. En défendant la spécificité du travail historien tout en l'assortissant d'une attention délicate aux ressources de la littérature, démarquant soigneusement le faux du fictif, la position de Ginzburg renvoie aux historiens une image plutôt flatteuse d'euxmêmes; aussi a-t-elle toutes les chances d'être adoptée comme leur ligne de défense.

Mais de défense contre quoi? Peut-être contre leur propre dilemme face à l'usage de la fiction comme outil théorique, qui constitue l'une des grandes tendances du moment. Dans le champ philosophique, Pierre Cassou-Noguès exploite la fiction narrative des philosophes qui "détermine le possible»: ainsi du cogito cartésien sortant tout armé de ce "roman de sciencefiction" que sont les Méditations métaphysiques ${ }^{44}$. On pourrait aussi citer des essais comparables en sociologie ou, dans le champ critique, le travail de Pierre Bayard qui consiste à fictionnaliser la fiction - par exemple en montrant tout le profit interprétatif qu'il y a à traverser "l'écran biographique" pour étudier la critique sociale dans "L'Étranger de Franz Kafka» ou ce grand roman russe qu'est "Autant en emporte le vent de Léon Tolstoï» ${ }^{45}$. L'historien rencontre quelque scrupule face à ce type de mensonge volontaire, même s'il n'en méconnaît pas l'efficacité lorsqu'il s'agirait de dépayser l'évidence, c'est-àdire de donner à voir ce point aveugle que constitue son propre point de vue, le lieu à partir duquel il discerne le spectacle du monde, mais dont il ignore tout sauf, précisément, à faire un pas de côté. C'est par exemple ce qu'avait tenté Daniel Milo pour rendre sensible l'artifice inaperçu de la découpe séculaire, en proposant une «histoire-fiction" qui consiste à décaler le siècle de trente-trois ans ( $\mathrm{Et}$ si on comptait à partir de la Passion?») pour produire une expérimentation: «De la science on emprunte la violence faite à l'objet étudié, c'est la définition même du laboratoire scientifique, de l'art d'avantgarde, l'idée que pour appréhender le réel et pour produire le beau -, il faut rendre le familier étrange, par la désautomatisation bien entendu ${ }^{46}$."

Seulement voilà : pour les historiens, toujours soumis, comme on l'a dit, au coup de froid théorique qui suivit la crise négationniste, le temps n'est plus au gai savoir un peu insouciant de ces expérimentations fictionnelles. Avis de tempête : chacun rentre au port. Que l'on songe, par exemple, à "l'histoire contre-factuelle" inspirée par la What if history américaine, qui se risque à décrire un passé qui n'a pas eu lieu à partir de l'incertitude de ses bifurcations. Les fortes réticences que rencontrent ces expériences d'uchronie témoignent clairement du fait que la virtualisation de l'histoire constitue une des limites du discours de l'historien ${ }^{47}$. Cette limite est peut-être aussi purement littéraire : rien ici ne peut égaler la puissance terrifiante de Philip Roth qui, dans Le Complot contre l'Amérique, fait d'un petit garçon apeuré le narrateur d'une histoire qui dérape - en 1940, Charles Lindbergh, héros de l'Amérique et antisémite notoire, est élu président des États-Unis ${ }^{48}$. C'est, une

43. C. Ginzburg, Le Fil et les traces, op. cit., p. 10.

44. Pierre Cassou-Noguès, Mon zombie et moi. La philosophie comme fiction, Éd. du Seuil, 2010, p. 74.

45. Pierre Bayard, Et si les ceuvres changeaient d'auteur?, Minuit, 2010.

46. Daniel S. Milo, Trahir le temps (histoire), Les Belles Lettres, 1991, p. 11.

47. Voir, par exemple, Anthony Rowley et Fabrice d'Almeida, Et si on refaisait l'histoire?, Odile Jacob, 2009.

48. Philip Roth, Le Complot contre l'Amérique [2004], trad. de l'anglais, Gallimard, 2006. 


\author{
Patrick Boucheron \\ On nomme littérature \\ la fragilité de l'histoire
}

fois de plus, par les moyens propres de la littérature que se révèle le caractère puéril du jeu ("on dirait que tu serais...») où s'exprime peutêtre l'inquiétante conjointure entre l'histoire et l'enfance ${ }^{49}$.

La tentation littéraire de l'historien

Il existe une forme commune du scrupule historien face à la littérature. C'est l'exergue. Elle est, dans l'usage ordinaire des exercices académiques, la réserve des regrets. On place en avant d'un livre, ou d'un article scientifique, un extrait littéraire, si possible imposant - à ce jeu, Claude Simon est l'un des plus fréquemment sollicités - ou ironiquement déplacé. Mais on laisse au lecteur le soin de découvrir par luimême les échos, subtils ou approximatifs, que cet éclat brillant entretient avec ce qui va suivre, inévitablement plus terne. Car ensuite, le texte historien vaque à ses affaires, comme libéré de ce qu'il aurait pu être ou n'a pas osé devenir. Parfois, ces regrets se disent explicitement. C'est le cas d'un livre où Lucette Valensi tente de reconstituer, de manière expérimentale, la vie d'un érudit juif de Tunis qui participa, à Trieste et surtout à Paris sous le Consulat, à l'aventure intellectuelle de l'orientalisme. Il s'ouvre par les premières lignes de La Chartreuse de Parme et s'achève par l'incipit de Lord fim qui campe fermement son personnage, mesurant sa taille, décrivant son allure, "ainsi s'ouvre Lord Fim, de Joseph Conrad et, une fois de plus, je l'envie ${ }^{50}$ ".

Mais que se passe-t-il lorsqu'un peu de littérature déborde de sa réserve et vient éclabousser la prose de l'histoire, et qu'appelle-t-on alors la tentation littéraire des historiens? On doit, me semble-t-il, l'écarter de leur envie romanesque. Que les historiens aient envie, par ailleurs, d'investir la scène littéraire est somme toute banal. La figure d'un professeur d'histoire qui écrit à ses moments perdus des romans policiers est une des modalités de cette double vie des écrivains qui caractérise aujourd'hui le jeu littéraire en France ${ }^{51}$. On pense évidemment ici aux romans de Fred Vargas qui n'entretiennent qu'un rapport distancié, ou parodique, avec sa profession d'historienne et d'archéologue médiéviste - le cas d'Umberto Eco, parvenant à convertir une renommée déjà impressionnante de sémiologue et d'historien du Moyen Âge en succès planétaire d'auteur de polars historiques qui, tout en devenant des best-sellers, ne renoncent à aucune de ses ambitions intellectuelles, est à tout point de vue exceptionnel.

On pourrait aussi évoquer le désir de théâtre qui taraude aujourd'hui les historiens, et qui s'exprimait peut-être déjà mezza voce dans l'envie que certains ont ressentie de reconstituer des dialogues fictifs, comme le fit Emmanuel Le Roy Ladurie lorsqu'il donnait l'illusion aux nombreux lecteurs de Montaillou qu'ils écoutaient aux portes des maisons d'un village haut perché de l'Ariège les conversations ordinaires des paysans qui les habitaient. Dans La Nuit blanche, Arlette Farge cherche plus explicitement "une forme d'écriture qui raconte et qui $\operatorname{expose}^{52}$ ». Son intention théâtrale est d'emblée politique ("prendre parti pour Pierre», exécuté à Cambrai en 1770 pour avoir envoyé des lettres

49. Giorgio Agamben, Enfance et histoire [1978], trad. de l'italien, Payot, 1989.

50. Lucette Valensi, Mardochée Naggiar. Enquête sur un inconnu, Stock, 2008, p. 332.

51. Bernard Lahire, La Condition littéraire. La double vie des écrivains, La Découverte, 2006. p. 9 .

52. Arlette Farge, La Nuit blanche, Éd. du Seuil, 2002, 
Patrick Boucheron

On nomme littérature

la fragilité de l'histoire anonymes qui troublèrent l'ordre public), comme l'est également celle de Gérard Noiriel ${ }^{53}$. On pense aussi à l'approche de Sophie Wahnich, empruntant à diverses formes d'intervention artistique (le livret d'opéra notamment), qui plaide pour une histoire sensible susceptible d'organiser la mise en partage politique des émotions et cherche le moyen de rendre le livre d'histoire "aussi déroutant ou aussi prenant qu'un roman contemporain, aussi fragile qu'une séquence de film, aussi dense qu'un recueil de poèmes, aussi drôle qu'une comédie réussie ${ }^{54}$ ».

Mais peu importent les genres, l'essentiel réside dans la déstabilisation de la langue ellemême. Il convient alors de reformuler la question: qu'est-ce qui peut bien pousser les historiens à mettre en péril les formes ordinaires de leur écriture académique? Celle-ci a des habitudes plus que des normes, des routines plus que des règles. Elles sont plus ou moins retorses. Quand on connaît, par exemple, l'histoire des notes de bas de page, qui ne trouve pas son origine dans les procédures d'administration de la preuve de l'histoire méthodique, mais dans les tours et détours du roman philosophique de la fin du xvirie siècle, où elles permettaient à l'auteur de faire retour, de manière volontiers satirique, sur son propre dire, on comprend mieux son fonctionnement rhétorique réel chez les historiens: leurs notes leur servent à la fois à objectiver leur discours et à affirmer de manière subreptice sa nature éminemment subjective ${ }^{55}$.

La neutralité de l'écriture académique est évidemment illusoire : l'historien écrit, s'engage tout entier dans l'acte même d'écrire, et il y a quelque naïveté scientiste à croire qu'il puisse exister un "langage commun des historiens ${ }^{56}$ " dans lequel ces derniers pourraient échanger leurs résultats de manière fiable et transparente. Et puisque l'historien écrit, il a seulement le choix entre deux possibilités : s'en désintéresser - et alors son texte fuitera en tout sens, de manière incontrôlée, se chargeant de messages subreptices (sur ses propres émotions, ses préjugés ou, pis encore, sur ceux de son temps, de son milieu, de ses engagements) qui sont les passagers clandestins de ses tours de langue, tapis inaperçus dans toute expression figée - ou s'en préoccuper.

On pense généralement que l'historien qui se préoccupe de son style souhaite faire le beau et se mettre en avant. S'il ne s'agit que de cela, si l'on veut seulement enjoliver son phrasé, alors les mots des historiens peuvent parader comme les notables de Flaubert aux comices agricoles: pompeux, rutilants, endimanchés. Certains s'y emploient. D'autres, au contraire, dès lors qu'ils s'expriment dans les journaux, croient nécessaire d'en adopter les tournures les plus viles (anachronismes faciles, œillades complices, effets appuyés, relâchement grammatical: tout ce registre de l'hypocorrection analysé par Pierre Bourdieu comme le privilège des puissants). Habitude détestable, mais qui peut sérieusement prétendre n'y avoir jamais cédé?

Dans les deux cas, il ne s'agit de rien d'autre qu'une adaptation anticipée à ce que l'on croit être les règles d'un marché éditorial clivé. L'exemple de Georges Duby permet pourtant

53. Gérard Noiriel, Histoire, théâtre, politique, Marseille, Agone, 2009

54. Sophie Wahnich, Les Émotions, la Révolution française et le présent. Exercices pratiques de conscience historique, CNRS, 2009.

55. Anthony Grafton, Les Origines tragiques de l'érudition. Une histoire de la note en bas de page, Éd. du Seuil, 1998.

56. Gérard Noiriel, Sur la "crise» de l'histoire, Belin, 1996, p. 203. 
Patrick Boucheron

On nomme littérature

la fragilité de l'histoire de comprendre comment sortir de ce faux dilemme. C'est lorsqu'il voulut élargir le type d'histoire qu'il croyait juste de défendre aux "nouvelles audiences ${ }^{57}$ " qu'ouvrait dans les années 1970 le développement de l'économie du livre qu'il entreprit de raffiner son écriture. Précisément parce que la matière historique était complexe et qu'il fallait se donner les moyens littéraires de l'offrir en partage en contrôlant ses effets. Mais dans le cas de Duby il est toujours important de rappeler - et les études menées actuellement dans la fabrique de son œuvre que documentent ses brouillons et dossiers préparatoires le confirment amplement - que le travail de l'écriture ne le divertissait jamais de son métier d'historien; au contraire, il l'y ramenait sans cesse ${ }^{58}$.

On peut chercher les moyens d'écrire autrement l'histoire sans nécessairement ressentir de l'hostilité, ou de l'insatisfaction, à l'égard des formes académiques de l'écriture savante. Sans doute faut-il même aujourd'hui en défendre la probité, l'exigence, la discrétion. Car elle est indispensable à la production des savoirs. En prenant soin d'elle, sans illusion, bien sûr, sur sa neutralité ou sa transparence, on accomplit certainement quelque chose comme un geste politique. Marc Bloch réclamait des historiens qu'ils sachent "parler du même ton aux doctes et aux écoliers ${ }^{59}$ ». La phrase est célèbre mais, au risque de paraître sacrilège devant ce saint laïc, figure tutélaire de la communauté des historiens, elle ne me semble pas pleinement satisfaisante. Avec la même exigence sans doute, mais pas du même ton ou en tout cas pas avec le même rapport à la langue commune - à ceci près qu'il est évidemment plus difficile de s'adresser aux écoliers qu'aux doctes : écrire mal, ou plus exactement ne pas se donner la peine d'écrire, est un luxe que l'on ne peut se permettre que lorsque l'on se contente de transmettre des informations dans le confort relâché de l'entre-soi - dès lors que l'on doit s'adresser en peu de mots à plus de gens, écrire est inévitable.

En cela, et en cela seulement, il existe un penchant des historiens que l'on peut dire littéraire - du moins si l'on entend bien la littérature comme une catégorie socialement construite, dont il existe évidemment mille définitions, mais qui peuvent s'accorder sur le fait qu'elle consiste à installer un rapport d'étrangeté dans sa propre langue. C'est, pour ma part, une interrogation dérangeante, à laquelle je ne connais pas de réponse : jusqu'où peut-on travailler sa langue tout en demeurant historien? Est-il un seuil proprement stylistique au-delà duquel se brise le pacte de croyance spécifique qui unit implicitement un livre d'histoire à ses lecteurs? Abandonner ses signes extérieurs de scientificité est une fausse audace : l'insurrection contre les notes de bas de page est sans risque aujourd'hui, de même qu'il est devenu assez courant d'adopter une mise en intrigue qui brise la linéarité du récit. Mais peut-on, par exemple, imaginer un texte d'histoire qui demeurerait tel tout en s'attaquant, non pas gratuitement mais pour des raisons proprement littéraires, à l'intégrité syntaxique de la phrase?

En écrivant l'histoire frêle et incertaine d'une centaine de Chinois que le régime mussolinien enferma en 1942 dans un camp des Abruzzes, Thomas Heams-Ogus réalise un projet qui correspond bien à ce que l'on pourrait attendre des historiens d'aujourd'hui. L'histoire de ces

57. Georges Duby, L'histoire continue, Odile Jacob, 1991, p. 125.

58. Voir, dans ce même numéro, la présentation de la première version, inédite, de son ego-histoire.

59. Marc Bloch, Apologie pour l'histoire ou Métier d'historien [1949], rééd. Armand Colin, 1997, p. 69. 
Patrick Boucheron

On nomme littérature

la fragilité de l'histoire prisonniers chinois ne peut s'achever à leur libération. "Où se coupe le fil ? Le fil ne se coupe pas. Il y a des dates, mais il y a des prolongements secrets ${ }^{60}$." Écrire ces prolongements, c'est mettre en péril la ligne claire du récit - et s'engager alors dans une aventure qui, nécessairement, dépaysera la langue commune. Que l'on songe par exemple, aujourd'hui, au travail extraordinairement précis qu'un écrivain comme Laurent Mauvignier exerce sur la phrase, ponctuée de saccades et de suspens, pour ne jamais Apprendre à finir. Débordant sans cesse du lieu d'un événement intime ou collectif qui a laissé Des hommes sans voix, il fait entendre le silence que la guerre d'Algérie a foré dans les âmes de ceux qui, l'ayant vécue, n'en parlent jamais. En ce sens, son projet ne diffère pas de celui des historiens, et encore récemment dans Ce que j'appelle oubli: Mauvignier écrit autour de la tache aveugle que constitue l'événement, non pour affronter de manière emphatique l'indicible, mais pour prêter l'oreille à ce qui se dit difficilement, à ce qui se dit tout de même, en employant un mot pour un autre ${ }^{61}$. Mais cet art littéraire est-il encore accessible aux historiens?

Écrire donc. Ce qui ne veut pas dire nécessairement "faire genre», en imitant les écrivains $\mathrm{du}$ moment. Je ne prendrais qu'un exemple, concernant l'histoire qui, on l'a vu, cristallise aujourd'hui l'inquiétude fictionnelle : celle de la Shoah. Le modèle de l'écrivain parti en quête d'une vie anonyme et engloutie est devenu si prégnant qu'il s'impose à l'historien. Au risque de la confusion. Dans un roman récent, Arnaud Rykner prétend écrire l'histoire du dernier train qui partit de Compiègne, le 2 juillet 1944, pour Dachau, et tente "cette chose obscène" (écrit-il) de prendre la place de celui qui s'est tu pour le faire parler : "Tout ce qui est raconté ici est vrai.
Tout ce qui est inventé ici est vrai aussi ${ }^{62}$." Dès lors qu'il s'inspire d'une telle démarche, l'historien se place au péril de l'indistinction fictionnelle. Par exemple, en tentant de reconstituer le destin d'une petite fille tuée à Auschwitz le 3 mars 1943, l'historien allemand Götz Aly est inévitablement amené à user de l'artifice romanesque qui consiste à combler les vides de l'histoire ${ }^{63}$.

C'est précisément cette posture que refusent Nicolas Mariot et Claire Zalc. Ils entendent renverser la perspective en brossant un portrait de groupe, à partir de l'ensemble des sources disponibles : celui des 991 juifs vivant à Lens entre 1940 et 1945. "Écrire l'histoire de ce groupe est notre ambition. L'écrire au ras du sol est notre choix ${ }^{64}$." Celui de la diversité des trajectoires individuelles pose le problème des dilemmes et des bifurcations : faut-il se déclarer ou fuir? partir ensemble ou se séparer? Le nombre permet ici de contourner l'une des difficultés de l'historien, face aux motivations des acteurs à faire ce qu'ils font. Ici, pas d'introspection psychologisante contrefaite, mais l'analyse froide des déterminations sociales qui pèsent sur les arbitrages de chacun. Froide, non, exacte, plutôt - et, au total, d'une grande noblesse dans un livre admirable de retenue. Ainsi l'historien offre-t-il ici aux morts ce qu'il a de plus précieux, au-delà de tout pathos: une morale de l'exactitude. Elle est menée

60. Thomas Heams-Ogus, Cent seize Chinois et quelques, Éd. du Seuil, 2010, p. 121

61. Laurent Mauvignier, Apprendre à finir, Minuit, 2000; Des hommes, Minuit, 2009; Ce que j'appelle oubli, Minuit, 2011.

62. Arnaud Rykner, Le Wagon, Rodez, Rouergue, 2010, p. 13.

63. Götz Aly, Into the Tunnel. The Brief Life of Marion Samuel, 1931-1943, New York, Metropolitan Books, 2007.

64. Nicolas Mariot et Claire Zalc, Face à la persécution. 991 Juifs dans la guerre, Odile Jacob, 2010, p. 8. 
Patrick Boucheron

On nomme littérature la fragilité de l'histoire avec d'autant plus de tact qu'elle connaît sa propre fragilité : le livre s'achève par l'irruption inopinée du $992^{\mathrm{e}}$ homme, Joseph Stohs, membre de l'équipe de football de Lens d'origine autrichienne qui, dans la nuit du 22 septembre 1942, fuit vers la Suisse. Échappant à la persécution, mais aussi, du même coup, à l'enregistrement documentaire qui la préparait et la précipitait, il était l'absent des fichiers, listes et recensements compulsés par les historiens.

La fragilité de l'histoire, justement

Ne pas combler les vides de l'histoire? L'historien peut crânement s'y refuser - d'autres le feront pour lui. Car la fiction l'emporte toujours, à la fin. Ainsi Pierre Senges se justifie-t-il de poursuivre les amorces de romans laissés inachevées par Kafka: «On aurait pu se contenter d'admirer ces restes tels qu'ils sont : malingres, pleins d'espérance - mais il existe deux lois irréfutables : 1) la nature a horreur du vide, 2) notre désir de récit est impossible à rassasier ${ }^{65}$." Faire profession d'historien consiste peut-être à admettre une fois pour toutes que l'on n'aura pas le dernier mot. Parce que la puissance de séduction du mensonge romanesque est irrépressible, l'historien a pour vocation de se faire contredire par plus ignorant que lui. Certes, il n'abdiquera pas pour autant toute ambition à se faire entendre. Lorsque les usages politiques de l'histoire en bousculent la probité de manière trop scandaleuse, il doit travailler à tenir son rang, à n'être point trop ridicule sur un plateau de télévision. Mais sans doute doit-il se résoudre à sa fragilité. Voyez cette histoire triste: on peinerait sans doute à trouver un historien qui a, plus qu'Alain Corbin, fait toute confiance à son propre talent littéraire pour enrichir les formes traditionnelles de l'écriture de l'histoire. Et de ce point de vue, Le Village des cannibales est certainement l'un de ses livres les plus inventifs, tenu de bout en bout par une sobriété de ton et une rigueur de composition qui maintiennent en alerte l'intérêt du lecteur, le laissant dans ses dernières lignes au bord de ce que peut l'histoire : «Seule reste à nu la cruauté, dans le ressac des sentiments ${ }^{66}$.» Or, dès lors qu'un romancier sympathique et télégénique a l'idée habile de s'emparer de la même histoire (le supplice à Hautefaye, en août 1870, d'un jeune aristocrate) pour en faire un roman mince et enlevé, il peut renvoyer cruellement le livre d'Alain Corbin dans le vrac d'une liste de "remerciements pour leur collaboration plus ou moins volontaire» où figurent à la fois Gustave Le Bon, René Girard et quelques érudits locaux - et personne n'y trouvera rien à redire, surtout pas les journalistes littéraires ${ }^{67}$. L'histoire, comme discipline et comme genre éditorial, y est alors ramenée à ses justes proportions: les plus illustres des historiens ne pèsent pas lourd face aux plus légers des romanciers.

Aussi faudrait-il pouvoir dire ceci : la tentation littéraire de l'historien est un aveu de faiblesse. Qui ne la voit? Cette faiblesse est partout: elle affecte évidemment le marché éditorial, et tout le malentendu vient du fait que l'on continue aujourd'hui à célébrer le triomphe littéraire des grands historiens de notre temps (Georges Duby en premier lieu) alors qu'il

65. Pierre Senges, Études de silhouette, Verticales, 2010, p. 9.

66. Alain Corbin, Le Village des cannibales, Aubier, 1990, p. 166. 2009. 
Patrick Boucheron

On nomme littérature

la fragilité de l'histoire correspond à une parenthèse enchantée de l'histoire de l'édition, refermée depuis longtemps. Surtout, la beauté de l'écriture ne servait pas à dire chez Duby les succès de l'historien, mais ses doutes et ses incertitudes. Sans doute se sont-ils approfondis aujourd'hui, du fait d'une cascade d'affaiblissements : elle affecte le rôle social de l'historien, concurrencé de plus en plus sévèrement par de nouveaux usages sociaux de l'histoire et de nouveaux magistères médiatiques; elle affecte les grands paradigmes explicatifs; elle affecte, surtout, le principe même de la transmission des savoirs mis à mal par la destruction politique de l'ancien monde universitaire.

Et puisqu'il convient peut-être, à la toute fin, de dévoiler un peu de ses intentions, les voici en quelques mots. J'appartiens à une génération qui ne se résoudra pas, du moins je l'espère, à s'abîmer dans la nostalgie d'un âge d'or qu'elle n'a connu que par ouï-dire. La crise de l'histoire? Au moins libère-t-elle des énergies qui font que, partout, malgré tout, on tente des choses. L'historien n'est plus l'instituteur du national et ne claironne plus son triomphe public? Je ne le regrette pas, préférant les voix plus profondes et plus délicates de ceux qui prennent soin des incertitudes de nos savoirs. Déclassements institutionnels, embarras mémoriels, désillusions éditoriales, débâcles universitaires : l'accumulation des échecs publics peut avoir comme conséquence paradoxale de libérer les historiens de leur arrogance, et j'avoue pour ma part que je les préfère ainsi, lorsqu'ils font moins les malins. J'ai tenté d'exprimer ailleurs ce désarroi joyeux, ce désenchantement qu'il convient de reconnaître non pour s'y complaire, mais pour y retrouver l'envie, pour moi inentamée, d'écrire un peu d'histoire ${ }^{68}$. Je tente de m'y employer en défendant à la fois la morale de l'exactitude et le débord de l'écriture, en faisant confiance au récit, à cette fiction qui ne ment pas, pour y fondre la méthode érudite qui l'autorise et la théorie qui l'inspire, tout en faisant le pari que l'une et l'autre y demeureront discernables ${ }^{69}$. Mais il y aurait quelque incongruité à théoriser après coup une pratique que je n'envisage pas autrement que comme un art d'exécution. Disons simplement qu'il ne peut être autre chose qu'un exercice de modestie, une ascèse de la netteté, un essai sensuel également - tout ce qui peut nommer la fragilité de l'histoire.

\section{Patrick Boucheron.}

68. Patrick Boucheron, Faire profession d'historien, Publications de la Sorbonne, 2010.

69. Id., Léonard et Machiavel, Lagrasse, Verdier, 2008. 